\title{
NUMERICAL SIMULATION OF A ELASTO-VISCOPLASTIC FLUID FLOW INSIDE A CAVITY
}

\author{
G. M. Furtado , \\ S. Frey ${ }^{a}$, \\ M. F. Naccache ${ }^{b}$, \\ ABSTRACT \\ This article addresses finite element approximations for elasto-viscoplastic \\ flows. Numerical simulations aiming at investigating the role of elasticity \\ for inertialess flows of viscoplastic materials within lid-driven cavity.The \\ mechanical model is made up of the usual governing equations for \\ incompressible fluids coupled with a Oldroyd-B type equation (de Souza \\ Mendes, 2011) modified to incorporate the dependency both of relaxation \\ and retardation time as the viscoplastic viscosity on the strain rate. These \\ parameters depend on the material microstructure, which level is described \\ by an structure parameter $\lambda$. This model is approximated by a multi-field \\ Galerkin least-squares formulation (Behr et al., 1993) in terms of extra- \\ stress tensor, the pressure field and the velocity vector. Results, focused on \\ the determination of yield surface topology, investigate the influence of \\ elastic and viscous governing parameters on the flow pattern. \\ Rio de Janeiro \\ giovannimf@mecanica.ufrgs.br

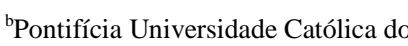 \\ Departamento de Engenharia Mecânica \\ Rio de Janeiro, RJ, Brasil \\ CEP 22453-900 \\ Received: April 11, 2018 \\ Revised: May 09, 2018 \\ Accepted: May 30, 2018 \\ Keywords: finite element, elasto-viscoplastic fuids, inestialess flows, \\ Oldroyd-B type, material microestructure
}

\section{NOMENCLATURE}

$\begin{array}{ll}\mathbf{D} & \text { strain rate tensor }\left(\mathrm{s}^{-1}\right) \\ \mathbf{f} & \text { bofy force }(\mathrm{N}) \\ \mathrm{L}_{\mathrm{c}} & \text { Characteristic length }(\mathrm{m}) \\ \mathrm{p} & \text { hydrostatic pressure }(\mathrm{Pa}) \\ \mathbf{u} & \text { velocity vector }(\mathrm{m} / \mathrm{s}) \\ \mathbf{x} & \text { coordinate vector }(\mathrm{m})\end{array}$

\section{Greek symbols}

$\alpha \quad$ stability parameter for momentum equation

$\gamma \quad$ strain rate, $\mathrm{s}^{-1}$

$\rho$ density, $\mathrm{kg} / \mathrm{m}^{3}$

$\tau \quad$ extra-stress magnitude $(\mathrm{Pa})$

$\tau$ extra-stress tensor $(\mathrm{Pa})$

$\mu \quad$ viscosity (Pa.s)

\section{Superscripts}

* dimensionless variables

\section{INTRODUCTION}

Elastic and viscous effects in the inertialess flow of elasto-viscoplastic fluids inside a lid-driven cavity are analyzed in the present work. The main feature of
non-Newtonian fluid presented in this work and that is present in a large variety of materials present in the petroleum industry, cosmetic and food products, is that this material has a behavior that depends on the value of the yield stress is below the stress initial flow. The main characteristic of the viscoplastic fluids is that below the "yield stress" they are highly structured materials with very high viscosities. When submmited to stresses above the yield limit their structure breaks, a deep viscosity decay occurs and they behave as purely viscous fluids, with constant or shear thinning viscosity.

Viscoplastic fluids can present elasticity and thixotropy. Recently, an Oldroyd-B type constitutive equation was proposed by (de Souza Mendes, 2009) to model the behavior of elasto-viscoplastic fluids, and also thixotropy. The equation involves the determination of a structure parameter to describe the fluid microstructure, with the aid of an aditional equation that has to be solved together with the conservation and constitutive equations.

Some works in the literature present numerical studies of viscoplastic fluid flows with no elasticity or thixotropy (e.g. (Alexandrou et al., 2001b); (Alexandrou et al., 2001a); (Alexandrou et al., 2001a); (Besses et al., 2003); (Burgos and Alexandrou, 1999); (Burgos et al., 1999); (Mitsoulis et al., 2006); (Naccache and Barbosa, 2007); 
(Mitsoulis et al.,1993); (Hammad et al., 2001); (Liu et al., 2002); (Zisis and Mitsoulis, 2002); and (Roquet and Saramito, 2003)). All these works neglected inertia effects, and employed regularized equations proposed by (Papanastasiou, 1987), by (Bercovier and Engelman, 1980) or by (de Souza Mendes and Dutra, 2004)) to model fluid viscosity. Regarding thixotropy and elasticity, there are not so many works that consider this kind of behavior either because modeling is still a challenge, or because of the lack of experimental data ((Barnes, 1997); (Mewis, 1979); (Mewis and Wagner, 2009), (de Souza Mendes and Thompson, 2012)). In a previous work, (Martins et al. 2013) analyzed the performance of a elastoviscoplastic fluid in the lid-driven cavity problem.

In this work, we solve the lid-driven cavity problem with the constitutive equation proposed by (de Souza Mendes, 2011), using a three-field Galerkin least-squares (GLS) finite element formulation. The formulation is able to successfully capture the elastic and viscous effects present in the current model, even making use of an equal-order combination of linear Lagrangian finite element interpolations. The role of elasticity, yield stress, shear-thinning and kinematics on the flow pattern are presented and discussed.

\section{MECHANICAL MODELING}

The mechanical model is written primarily by the conservation equations of mass and momentum, given by:

$$
\begin{gathered}
\operatorname{div} u=0 \operatorname{in} \Omega \\
\rho(\nabla u) u+p-\operatorname{div} \tau-f=\operatorname{din} \Omega
\end{gathered}
$$

where $\boldsymbol{u}$ is velocity vector, $P=p+\rho \phi$ is the modified pressure, $p$ is the pressure, $\rho$ is the fluid density, and is the $g=-\nabla \phi$ is the gravitational force per unit mass.

The constitutive equation of the model adopted in this work for the extra-stress tensor $\tau=T+p 1$ is given by:

$$
\tau+\theta_{1} \stackrel{\nabla}{\tau}=\eta\left(\dot{y}+\theta_{2} \gamma\right) \operatorname{in} \Omega
$$

Where $\dot{y}=\nabla u+\nabla u^{t}$ is the rate of deformation tensor field, and the upper-convected time derivatives of $\tau$ and $\dot{y}$ are given by:

$$
\tau=\frac{d \tau}{d t}-\tau . \nabla u-\nabla u^{T} \cdot \tau
$$

$$
\stackrel{\dot{\nabla}}{\gamma}=\frac{d \dot{\gamma}}{d t}-\dot{\gamma} \cdot \nabla u-\nabla u^{T} \cdot \dot{\gamma}
$$

where $\mathrm{d}(*) / \mathrm{dt}=\partial(*) / \partial t+u . \nabla(*)$ is the material time derivative.

In this model, its rheological parameters, viscosity function $(\eta)$, relaxation time $\theta_{1}=\left(1-\frac{\eta_{\infty}}{\eta_{e q}}\right) \frac{\eta_{e q}}{G_{e q}}$ and $\quad$ retardation time $\theta_{2}=\left(1-\frac{\eta_{\infty}}{\eta_{e q}}\right) \frac{\eta_{\infty}}{G_{e q}}$ are written in terms of a parameter structure $\lambda$, which evolves with the time that the fluid is being submitted to the certain stress. This structure parameter is time dependent, we can just assign it to a thixotropic behavior. In the model used in the present work, the evolution equation for $\lambda$ is given by:

$$
\begin{aligned}
& \frac{d \lambda}{d t}=\frac{\partial \lambda}{\partial t}+ u \cdot \nabla(\lambda)= \\
& \frac{1}{t_{e q}}\left[(1-\lambda)-\left(1-\lambda_{e q}\right)\left(\frac{\lambda}{\lambda_{e q}}\right)\right]
\end{aligned}
$$

where the RHS is composed of a (positive) buildup term and a (negative) breakdown term de Souza Mendes (2009).

The parameter $t_{e q}$ is the equilibrium time, which physically means a time scale for the microstructure buildup process. It can be easily observed that when $t_{e q} \rightarrow 0$ the fluid structure instantaneoulsy develops to its equilibrium state and no thixotropic behavior is observed. This situation result in a purely elasto-viscous behavior, and is the situation analyzed in the present work. The relation between the structure parameter and the equlibrium viscosity is given by:

$$
\lambda_{e q}(\dot{y})=\frac{\ln \eta_{e q}(\dot{y})-\ln \eta_{\infty}}{\ln \eta_{0}-\ln \eta_{\infty}}
$$

where $\eta_{0}$ is the zero-shear-rate viscosity and $\dot{y}=\sqrt{1 / 2 \operatorname{tr}^{2}}$ is the intensity of $\dot{y}$. The equilibrium structure parameter $\dot{y}_{e q}$ is a scalar quantity that varies within the range $[0,1]$. It gives a measure of the structuring level of the microstructure, i.e., $\lambda_{e q}=0$ when the structuring level is minimum, and $\lambda_{\text {eq }}=1$ when the material is fully structured. 
Therefore, the $\lambda_{\text {eq }}$ can thus be seen as a normalized equilibrium viscosity function, since there is a one-toone relationship between structure and viscosity levels.

As seen, the relaxation time and retardation time are written in terms of the infinite-shear-rate viscosity $\eta_{\infty}$, the equilibrium viscosity $\eta_{e q}$ and the equilibrium elastic modulus (de Souza Mendes and Thompson, 2012b), given by:

$$
G_{e q}=G_{0} e^{m\left(\frac{1}{\lambda_{e q}}-1\right)}
$$

where $G_{0}$ is the structural elastic modulus of the fully structured material, and $m$ is a positive scalar parameter that dictates the sensitivity of $G_{e q}$ with $\lambda_{e q}$. In this equation, it can be observed that its lowest value (i.e., highest relaxation and retardations times) occurs at the highest value of $\lambda$, and it increases as $\lambda$ decays. This function is chosen to simulate the elastic behavior of viscoplastic fluids at the regions of low stress values, i.e., where the stress is below the yield stress.

In this work, we employed the following expression for the equilibrium viscosity (de Souza Mendes and Dutra, 2004):

$$
\begin{aligned}
& \eta_{e q}(\dot{y})= \\
& {\left[1-\exp \left(\frac{-\eta_{0} \dot{y}}{\tau_{y}}\right)\right]\left\{\frac{\tau_{y}}{\dot{y}}+K \dot{y}^{n-1}\right\}+\eta_{\infty}}
\end{aligned}
$$

where $\tau_{y}$ is the yield stress, $K$ the consistency index, and $n$ the power-law index.

The fluid behavior is characterized by three different regions in the flow curve, namely the end of the highest viscosity plateau $\dot{y}_{0}$, the begining of the power law viscosity behavior $\dot{y}_{1}$ and the begining of the lowest viscosity plateau $\dot{y}_{2}$, respectively:

$$
\dot{y}_{0}=\frac{\tau_{y}}{\eta_{0}}, \dot{y}_{1}=\left(\frac{\tau_{y}}{K}\right)^{1 / n}, \dot{y}_{2}=\left(\frac{\eta_{\infty}}{K}\right)^{1 / n-1}
$$

where $\eta_{0}$ is the low shear rate viscosity plateau $\tau_{y}$,is the yield stress, $K$ is the consistency index, $n$ the power-law index and $\eta_{\infty}$ is the high shear rate viscosity plateau.

The governing dimensionless equations for nonthixotropic, steady creeping flows are given by:

$$
\begin{gathered}
t^{*}=t \dot{y}_{1}, x^{*}=\frac{x}{L}, u^{*}=\frac{u}{\dot{y}_{1} L}, \dot{y}^{*}=\frac{\dot{y}}{\dot{y}_{1}} \\
P^{*}=\frac{P}{\tau_{y}}, \tau^{*}=\frac{\tau}{\tau_{y}}, \eta_{e q}^{*}=\frac{\eta_{e q} \dot{\gamma}_{1}}{\tau_{y}}
\end{gathered}
$$

where the superscript $*$ indicates that the variable is dimensionless. Using these definitions, the dimensionless versions of the governing equations for steady creeping flows are given by:

$$
\begin{gathered}
\nabla^{*} \cdot u^{*}=0 i n \Omega^{*} \\
\rho\left(\nabla^{*} u^{*}\right) u^{*}+\nabla^{*} p^{*}-\operatorname{div} \tau^{*}-f^{*}=0 i n \Omega^{*} \\
\left.\tau^{*}+\theta_{1}^{*}(\lambda) \tau^{*}=2 \eta_{v}^{*}(\lambda)\left[\dot{\gamma}^{*}+\theta_{2}^{*} \gamma\right] \operatorname{\nabla }\right] \Omega^{*} \\
\lambda=\lambda_{e q} i n \Omega^{*}
\end{gathered}
$$

where, $\theta_{i}^{*}(\lambda)=\theta_{i}(\lambda) \dot{\gamma}_{1}$ the dimensionless relaxation time, $\dot{\gamma}^{*}=\frac{1}{2}\left(\nabla^{*} u^{*}+\nabla^{*} u^{* T}\right), \quad$ and $\tau^{*}=\left(\nabla^{*} \tau^{*}\right) \cdot u^{*}-\left(\nabla^{*} u^{*}\right) \cdot \tau^{*}-\tau^{*} \cdot\left(\nabla^{*} u^{*}\right)^{T}$. The dimensionless viscosity, relaxation and retardation times are given by, respectively:

$$
\begin{aligned}
& \eta_{e q}^{*}\left(\dot{\gamma}^{*}\right)= \\
& {\left[1-\exp \left(-(J+1) \dot{\gamma}^{*}\right)\right]\left(\frac{1}{\dot{\gamma}^{*}}+\dot{\gamma}^{* n-1}\right)+\eta_{\infty}^{*}} \\
& \theta_{1}^{*}\left(\dot{\gamma}^{*}\right)=\alpha \frac{\eta_{e q}^{*}}{G_{0}^{*}}, \theta^{*}{ }_{2}\left(\dot{\gamma}^{*}\right)=\alpha \frac{\eta_{\infty}^{*}}{G_{0}^{*}}
\end{aligned}
$$

where

$$
\alpha=\frac{\left(1-\eta_{\infty}^{*}\right)}{\exp \left(m\left(1 / \lambda_{e q}-1\right)\right)}
$$

The following governing parameters may be identified from the above equations:

$$
J=\frac{\dot{y}_{1}-\dot{y}_{0}}{\dot{y}_{0}}=\eta_{0}\left(\frac{\tau_{y}^{1 / n-1}}{K^{1 / n}}\right)-1
$$




$$
U^{*}=\frac{u_{c}}{\dot{\gamma}_{1} L}
$$

where the dimensionless velocity $U^{*}$ arises from the boundary condition at the cavity lid, which controls the flow intensity inside the cavity. The parameter $J$ gives a relative measure of the colapse of the material microstructure (see de Souza Mendes, 2007 for more details).

\section{Numerical Approximation}

To approximate the mechanical model described above it was employed a multi-field stabilized Galerkin least-squares formulation in therms of velocity, pressure and extra-stress. The classical Galerkin method does not guarantee stable approximations, may generate solutions without physical meaning and numerical pathologies for mixed incompressible fluid flows. The inherent difficulties associated to the Galerkin method are due to the compatibility of velocity and pressure finite element subspaces, e.g., the need to satisfy the Babuška-Brezzi condition involving these subspaces, a condition which was established by Babuška and Brezzi in the early 70's. The velocity and pressure subspaces may not be spanned by any arbitrary combination of finite element interpolations and, in the case of this work, which employs a multi-field formulation, another compatibility condition must be imposed on the choice of the stress and velocity subspaces. The alternative to remedy Galerkin deficiencies adopted here for incompressible fluid flows was to change the classical Galerkin formulation - adding mesh-dependent terms, which are functions of the residuals of flow governing equations, evaluated element-wise - and use simple Lagrangean elements.

The Fig. 1 schematically shows the $L_{c} \times L_{c}$ geometry with the employed boundary conditions: uniform unitary velocity in the $x_{2}$ direction on the top wall and non-slip condition $(u=0)$ at the remaining walls. For all computations, it is used a mesh with 10000 elements, with 10201 nodal points.

A mesh independence procedure evaluating the relative error of the extra-stress magnitude is performed. The Fig. 2 shows a detailed view of the stress profile at $x_{1}^{*}=0.5$. Despite results are almost coincident for the meshes investigated (errors below $3 \%$ ), the more refined mesh tested - with 100 Q1 finite elements and 10,201 nodal points, which produces a smallest value of its non-dimension element size $h_{K_{\min }}^{*}=h_{K} / L=1.41 .10^{-2}$ is selected in order to guarantee more accurate approximations close the cavity corners.



Figure 1. The geometry.

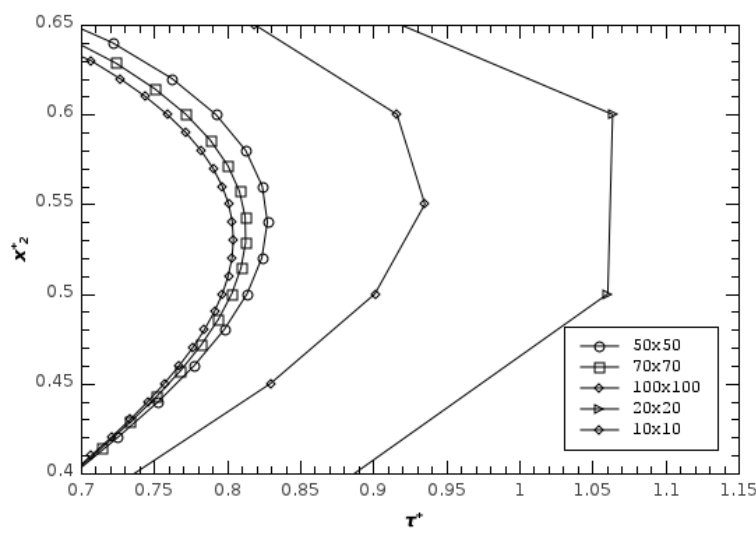

Figure 2. The independence mesh.

\section{Numerical results}

All the simulations are obtained for steady flows, neglecting inertia and thixotropy $t_{e q}^{*} \rightarrow 0$, i.e., the material structure changes imediately after being submitted to a certain stress level. Also, all the results are obtained for negligible retardation times. The results show the effects of the rheological parameters and of the lid cavity velocity on the yield surfaces. The yield surfaces are defined as the locus of points in which the magnitude of the strain rate is below the lowest shear rate value for which the viscosity equals the higher viscosity plateau where $\eta=\eta_{0}$, i.e., when, $\dot{y}<\dot{y}_{0}$ (dos Santos et al., 2011).

All the results are obtained for $\eta_{\infty}^{*}=0.01$.

The Figure 3 show the influence of the elasticity of yield surfaces. The black regions represent the unyielded zones $\dot{y}<\dot{y}_{0}$, while the white regions represent the yielded zones. As fluid elasticity increases, it can be observed that there are two unyielded zones, one symmetric (excepet Fig.3.(d)), attached to the bottom wall and another one, nonsymmetric, closer to the upper wall, located in the core of a flow recirculating zone. Elasticity is 
stronger inside the unyielded regions. Its effect is to increase the stress levels and the deformation rates, which in turn reduces the size of the unyielded regions.
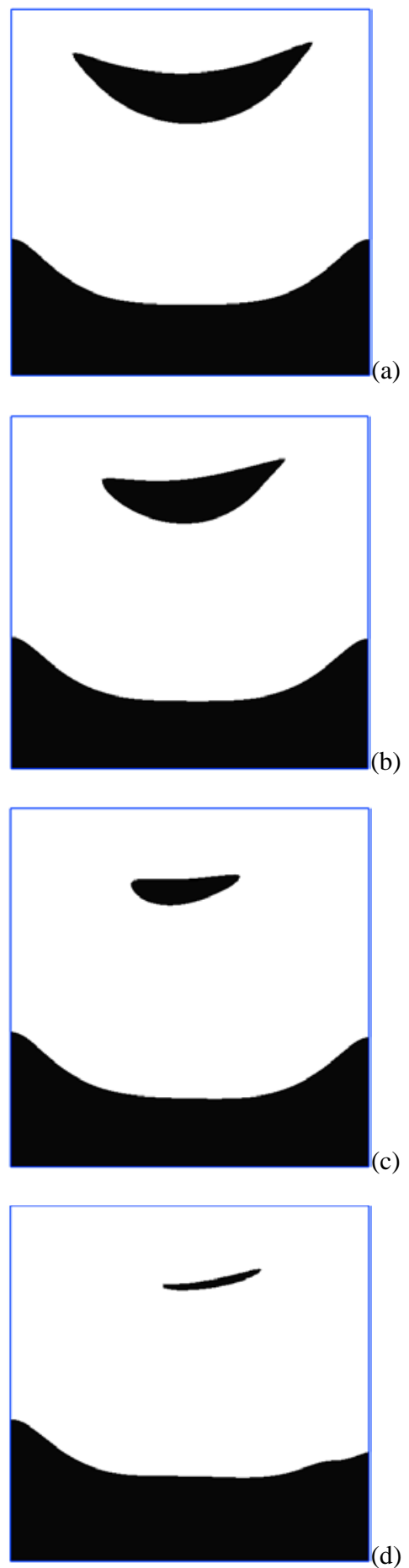

Figure 3. Yield surfaces: effect of the elasticity: $J=1000, n=0.5, U^{*}=0.05$, (a) $G_{0}^{*}=100$;
(b) $G_{0}^{*}=20$
(c) $G_{0}^{*}=5$;
(d) $G_{0}^{*}=1$.

The effect of the lid cavity velocity is shown in Fig. 4 for $J=1000, n=0.5$, and $G_{0}^{*}=10$. As expected, increasing the lid velocity (which is equivalent to decrease the yield stress) leads to smaller (upper and bottom) apparently unyielded regions due to higher levels of velocities and consequently, higher deformation rates as well. Again, due to elasticity (recall that there is no inertia), the flow pattern is non symmetric and the stress and strain levels are higher.
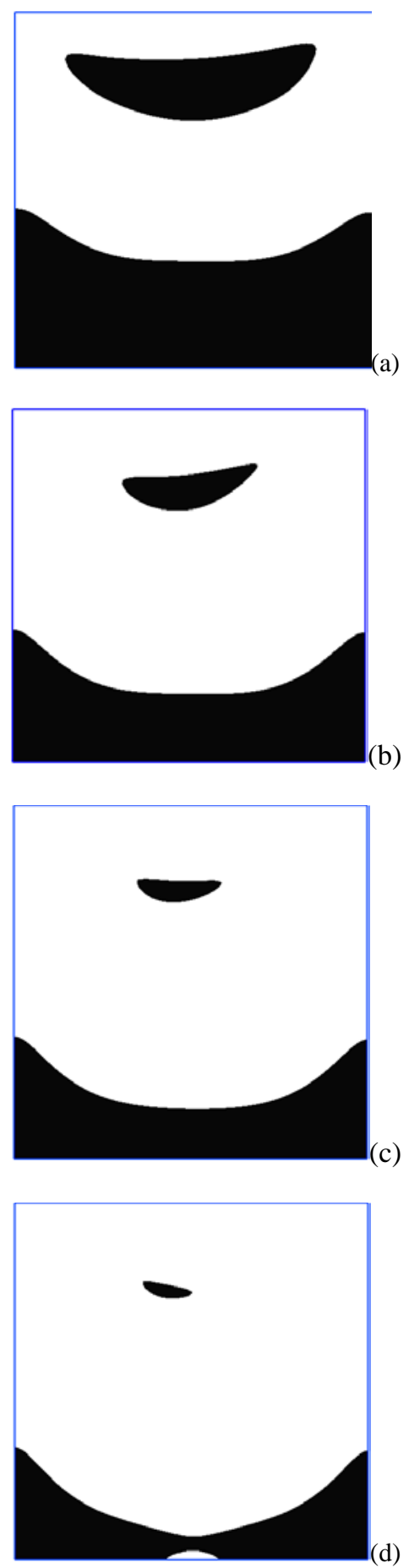

Figure 4. Yield surfaces: effect of lid velocity for: $G_{0}^{*}=10, n=0.5$ and $J=1000$, (a) $U^{*}=0.01$;

(b) $U^{*}=0.05$; (c) $U^{*}=0.1$; (d) $U^{*}=0.2$. 
The influence of the elasticity in the elastic strain is show in the Figure 5. The highest values of elastic strain are near from apparently unyielded regions, since the strain rate within the apparently unyielded regions is low. According to the increase in elasticity, the field of elastic deformation increases within the cavity.
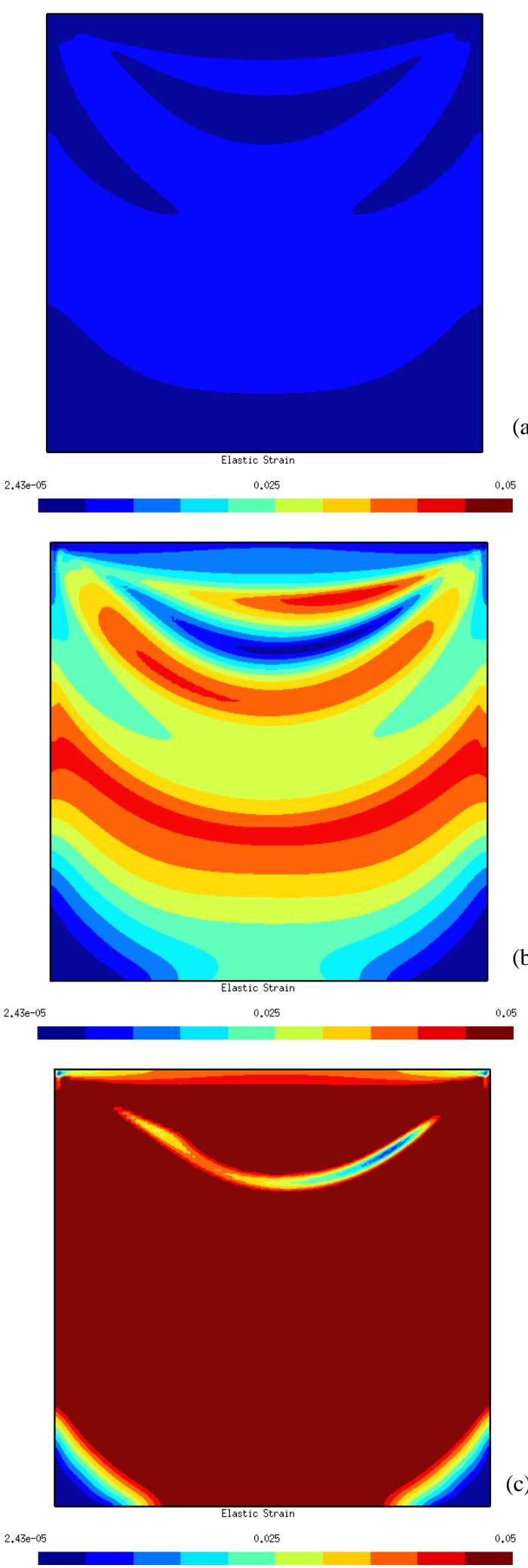

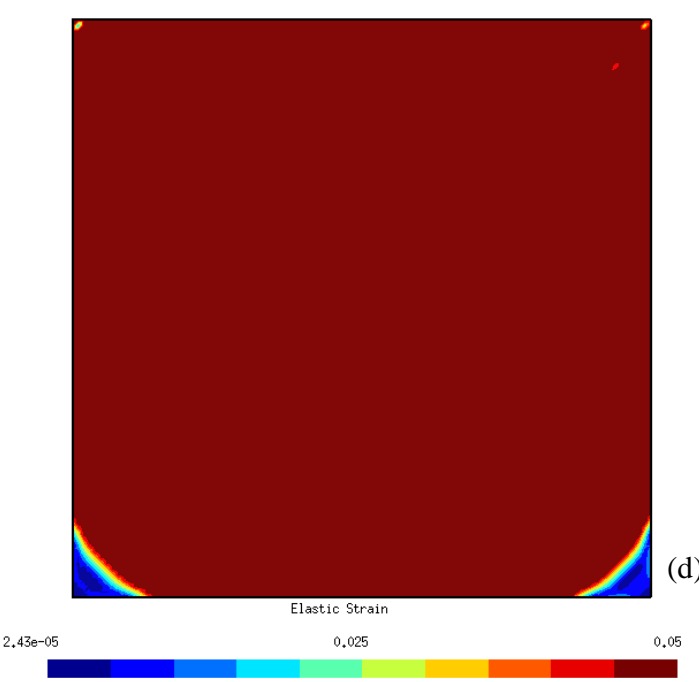

Figure 5. Elastic Strain for: $J=1000, n=0.5$

$$
\begin{aligned}
\text { and } U^{*}= & 0.05 \text {, (a) } G_{0}^{*}=100 \text {, (b) } G_{0}^{*}=20 \text {; } \\
& \text { (c) } G_{0}^{*}=5 \text {; (d) } G_{0}^{*}=1 .
\end{aligned}
$$

\section{CONCLUSIONS}

The numerical simulation, besides the usual viscous effects on the morphology and location of unyielded regions, confirm the relevant role of elasticity for an accurate determination of yield surfaces, namely, the monotonic increase of elasticity gives rise to more and more asymmetric yield surfaces. And from a certain velocity, as shown in Figure 4 (d), happens a separation of apparently unyielded regions because the higher the speed, the inertia effect is stronger.

\section{ACKNOWLEDGEMENTS}

S. Frey, M. F. Naccache and P. R. de Souza Mendes acknowledges CAPES and $\mathrm{CNPq}$ for financial support.

\section{REFERENCES}

Alexandrou, A. N., and Entov, V., 2001, Inertial, Viscous and Yield Stress Effects in Bingham Fluid Filling of a 2-D Cavit, Journal of NonNewtonian Fluid Mechanics, Vol. 96, pp. 383-403.

Alexandrou, A. N., and Burgos, G., 2001, Steady Herschel-Bulkley Fluid Flow in ThreeDimensional Expansions, Journal of Non-Newtonian Fluid Mechanics, Vol. 100, pp. 77-96.

Barnes, H. A., 1997, Thixotropy - A Review, Journal of Non-Newtonian Fluid Mechanics, Vol. 70, pp. 1-33.

Barnes, H. A., 1999, A Brief History of the Yield Stress, Applied Rheology, Vol. 9, pp. 262-266.

Barnes, H. A., 1999, The Yield Stress - A Review, Journal of Non-Newtonian Fluid Mechanics, Vol. 81, pp. 133-178. 
Bercovier, M., and Engelman, M., 1980, A Finite-Element Method for Incompressible NonNewtonian Flows, Journal of Computational Physics, Vol. 36, pp. 313-326.

Besses, D. D. D., Magnin, A., and Jay, P., 2003, Viscoplastic Flow Around a Cylinder in an Infinite Medium, Journal of Non-Newtonian Fluid Mechanics, Vol. 115, pp. 27-49.

Burgos, G. R., and Alexandrou, A. N., 1999, Flow Development of Herschel-Bulkley Fluids in a Sudden 3-D Expansion, Journal of Rheology, Vol. 43, No. 3, pp. 485-489.

Burgos, G., Alexandrou, A. and Entov, V., 1999, On the Determination of Yield Surfaces in Herschel-Bulkley Fluids, Journal of Rheology, Vol. 43, No. 3, pp. 463-483.

Carter, R. E., and Warren, R. C., 1987, Extrusion Stresses, Die Swell, and Viscous Heating Effects in Doublebase Propellants, Journal of Rheology, Vol. 31, pp. 151-173.

de Souza Mendes, P. R., 2007, Dimensionless Non-Newtonian Fluid Mechanics, Journal of NonNewtonian Fluid Mechanics, Vol. 147, pp. 109-116. de Souza Mendes, P. R., 2009, Modeling the Thixotropic Behavior of Structured Fluids, Journal of Non-Newtonian Fluid Mechanics, Vol. 164, pp. 6675.

de Souza Mendes, P. R., 2011, Thixotropic Elasto-Viscoplastic Model for Structured Fluids, Soft Matter, Vol. 7, pp. 2471-2483.

de Souza Mendes, P. R., and Dutra, E. S. S., 2004, Viscosity Function for Yield-Stress Liquids, Applied Rheology, Vol. 14, No. 6, pp. 296-302.

de Souza Mendes, P. R., Naccache, M. F., Varges, P. R. and Marchesini, F. H., 2007, Flow of Viscoplastic Liquids through Axisymmetric Expansions Contractions, Journal of Non-Newtonian Fluid Mechanics, Vol. 142, No. 1-3, pp. 207-217.

de Souza Mendes, P. R., and Thompson, R. L., 2012, A Critical Overview of Elasto-Viscoplastic Thixotropic Modeling, Journal of Non-Newtonian Fluid Mechanics, Vol. 187, pp. 8-15.

dos Santos, D., Frey, S., Naccache, M., and de Souza Mendes, P., 2011, Numerical Approximations for Flow of Viscoplastic Fluids in a Lid-Driven Cavity, Journal of Non-Newtonian Fluid Mechanics, Vol. 166, pp. 667-679.

Hammad, K. J., Vradis, G. C., and Ötügen, M. V., 2001, Laminar Flow of a Herschel-Bulkley Fluid Over an Axisymmetric Sudden Expansion, Journal of Fluids Engineering, Vol. 123, pp. 588-594.

Liu, B. T., Muller, S. J., and Denn, M. M., 2002, Convergence of a Regularization Method for Creeping Flow of a Bingham Material about a Rigid Sphere, Journal of Non-Newtonian Fluid Mechanics, Vol. 102, pp. 179-191.

Martins, R. R., Furtado, G. M., dos Santos, D. D., Frey, S., Naccache, M. F., and de Souza Mendes, P. R., 2013, Elastic and Viscous Effects on Flow Pattern of Elasto-Viscoplastic Fluids in a Cavity,
Mechanics Research Communications, Vol. 53, pp. 36-42.

Mewis, J., 1979, Thixotropy - A General Review, Journal of Non-Newtonian Fluid Mechanics, Vol. 6, pp. 1-20.

Mitsoulis, E., Abdali, S. S., and Markatos, N. C., 1993, Flow Simulation of Herschel-Bulkley Fluids through Extrusion Dies, Journal of NonNewtonian Fluid Mechanics, Vol. 71, pp. 147-160.

Mitsoulis, E., Marangoudakis, S., Spyratos, M., and Zisis, T., 2006, Pressure-Driven Flows of Bingham Plastics over a Square Cavity, Journal of Fluids Engineering, Vol. 128, pp. 993-1003.

Naccache, M. F., and Barbosa, R. S., 2007, Creeping Flow of Viscoplastic Materials through a Planar Expansion Followed by a Contraction, Mechanics Research Communications, Vol. 34, pp. 423-431.

Nassar, B., de Souza Mendes, P. R., and Naccache, M. F., 2011, Flow of Elasto-Viscoplastic Liquids through an Axisymmetric ExpansionContraction, Journal of Non-Newtonian Fluid Mechanics, Vol. 166, pp. 386-394.

Papanastasiou, T. C., 1987, Flows of Materials with Yield, Journal of Rheology, Vol. 31, pp. 385404.

Roquet, N., and Saramito, P., 2003, An Adaptive Finite Element Method for Bingham Fluid Flows around a Cylinder, Computer Methods in Applied Mechanics and Engineering, Vol. 192, pp. 3317-3341.

Saramito, P., 2007, A New Constitutive Equation for Elastoviscoplastic Fluid Flows, Journal of Non-Newtonian Fluid Mechanics, Vol. 145, pp. 114.

Sikorski, D., Tabuteau, H., and de Bruyn, J. R., 2009, Motion and Shape of Bubbles Rising through a Yield-Stress Fluid, Journal of Non-Newtonian Fluid Mechanics, Vol. 159, pp. 10-16.

Zisis, T., and Mitsoulis, E., 2002, Viscoplastic Flow around a Cylinder Kept between Parallel Plates, Journal of Non-Newtonian Fluid Mechanics, Vol. 105, pp. 1-20. 